\section{La prevención de la muerte súbita en pacientes con enfermedad coronaria}

En años recientes se ha observado un descenso de las tasas de mortalidad por enfermedades cardiovasculares. No obstante, la mortalidad sigue siendo alta en pacientes que han sufrido infarto agudo del miocardio y que salen del hospital con lesión extensa del ventrículo izquierdo y arritmias ventriculares asintomáticas, pese al tratamiento con fármacos antiarrítmicos. En este grupo, la mortalidad al cabo de $4-5$ años es quizá superior a $20 \%$ y una tercera parte de esta mortalidad tardía se compone de casos de muerte súbita. Pese a la existencia de pautas muy concretas para el tratamiento de pacientes que sufren un paro cardíaco fuera del hospital, solamente 2 a $30 \%$ de estos pacientes sobreviven. Por consiguiente, se impone la necesidad de mejorar las medidas para la prevención del paro cardíaco.

Según los resultados de algunos estudios, se puede reducir el riesgo de muerte súbita si el tratamiento antiarrítmico se combina con pruebas electrofisiológicas orientadoras. A fin de explorar esta hipótesis, un grupo de investigadores llevó a cabo un estudio controlado aleatorizado en 704 pacientes con enfermedad coronaria, una fracción de expulsion ventricular izquierda ${ }^{1}$ máxima de $40 \%$ y episodios esporádicos de taquicardia ventricular asintomática. Los pacientes, en quienes se provocaron taquiarritmias ventriculares con estímulos programados, fueron asignados aleatoriamente a uno de dos grupos: uno recibió tratamiento antiarrítmico con fármacos y desfibriladores implantables y el otro no fue sometido a ningún tratamiento. Se administraron inhibidores de la enzima convertidora de la angiotensina y bloqueantes de los receptores beta adrenérgicos a aquellos pacientes que pudieron tolerar estos medicamentos.

Según el método de estimación de KaplanMeier, la incidencia de paro cardíaco o de muerte por arritmia fue de $25 \%$ en los pacientes cuyo tratamiento con antiarrítmicos fue dirigido por los resultados de pruebas electrofisiológicas; en cambio, dicha incidencia fue de $32 \%$ entre los pacientes que no recibieron ningún tratamiento antiarrítmico

\footnotetext{
$1100 \times$ ([volumen ventricular al final de la diástole - volumen ventricular al final de la sístole $] \div$ volumen ventricular al final de la diástole).
}

(riesgo relativo [RR]: 0,73; IC95\%: 0,53 a 0,99), lo cual equivale a una reducción de $27 \%$. La mortalidad estimada al cabo de 5 años en ambos grupos fue de 42 y $48 \%$, respectivamente (RR: 0,80; IC95\%: 0,64 a 1,01). El riesgo de paro cardíaco o de muerte por arritmias en los pacientes con desfibriladores implantables fue significativamente menor que en pacientes que salieron del hospital sin haber recibido un desfibrilador (RR: 0,24; IC95\%: 0,13-0,45; $P<0,001)$. En cambio, no hubo ninguna reducción detectable de la frecuencia de paro cardíaco o de la mortalidad general en pacientes tratados con medicamentos antiarrítmicos con la orientación de pruebas electrofisiológicas, al compararse con los pacientes que no recibieron tratamiento alguno.

En resumen, el uso de pruebas electrofisiológicas para orientar el tratamiento reduce el riesgo de muerte súbita en pacientes con enfermedad coronaria extensa cuando son tratados con desfibriladores implantables, pero no parece aportar ningún beneficio en pacientes cuyo tratamiento se limita al uso de fármacos antiarrítmicos. (Buxton $\mathrm{AE}$, Lee KL, Fisher JD, Josephson ME, Prystowsky EN, Hafley $\mathrm{G}$, et al. A randomized study of the prevention of sudden death in patients with coronary artery disease. New Engl J Med 1999;341(25):18-1890).

\section{La mortalidad y el peso corporal en personas que nunca han fumado}

Numerosos estudios prospectivos han revelado una asociación entre el exceso de peso corporal y la mortalidad en adultos, pero pocos han explorado la posibilidad de que un adulto sano con un peso corporal demasiado bajo también esté en mayor riesgo de morir que una persona de peso normal. Este interrogante cobra especial pertinencia a la luz de la reciente reducción de 21 a 19 del límite inferior del índice de masa corporal (IMC $=$ [peso $(\mathrm{kg}) /$ estatura $\left.\left(\mathrm{m}^{2}\right)\right]$ ) recomendado para adultos estadounidenses por los Institutos Nacionales de Salud, de Bethesda, Maryland.

La mayor mortalidad que se viene observando desde hace 30 años en adultos de muy bajo peso corporal en muchas poblaciones se ha atribuido al efecto de factores de confusión, particularmente el tabaquismo y la presencia de enfermedades subyacentes. No obstante, algunos estudios prospectivos recientes en pacientes sanos que nunca habían fu- 
mado indican que el riesgo de mortalidad en individuos con un IMC muy bajo es similar al del resto de la población, aunque se observan diferencias según la edad y el sexo.

En un estudio prospectivo de 12 años de duración, un grupo de investigadores examinó la relación entre el IMC y la mortalidad. La población estudiada, derivada del Estudio de Salud Adventista que se llevó a cabo en California (1976-1988), se compuso de 20346 adultos blancos no hispanos (divididos en dos grupos: adultos de mediana edad, de 25-54 años; adultos de edad avanzada, de 55-84 años) que nunca habían fumado y que no tenían antecedentes de enfermedad coronaria, cáncer o accidente cerebrovascular. Se buscaron indicadores de enfermedad subyacente (peso actual comparado con el que se tenía 17 años antes; defunción durante la etapa inicial del seguimiento) y se halló una asociación positiva directa entre el IMC y la mortalidad por todas las causas en hombres de mediana edad (riesgo mínimo con un IMC de 15-22,3), hombres de edad avanzada (riesgo mínimo con un IMC de 13,5-22,3), mujeres de mediana edad (riesgo mínimo con un IMC de 13,9-20,6) y mujeres de edad avanzada (riesgo mínimo con un IMC de 13,4-20,6). En mujeres posmenopáusicas que no habían recibido tratamiento hormonal de reemplazo los autores encontraron una relación en forma de $\mathrm{J}$ (riesgo mínimo con un IMC de 20,7-27,4), con un riesgo doble de defunción (riesgo relativo: 2,2; IC95\%: 1,3 a 3,5) en mujeres de edad avanzada cuyo IMC era $<20,7$, principalmente por enfermedades cardiovasculares y respiratorias. En cambio, este tipo de asociación no se observó en mujeres adultas de menor edad, como tampoco en el sexo masculino.

Los resultados del presente estudio apuntan a la posibilidad de un riesgo más alto de mortalidad en mujeres mayores que tienen un IMC excesivamente bajo, quizá debido a la escasa síntesis de los estrógenos que normalmente se producen en el tejido adiposo por un proceso de aromatización, a la cual se suma la carencia producida por el cese de la función ovárica. (Singh PM, Lindsted KD, Fraser GE. Body weight and mortality among adults who never smoked. Am J Epidemiol 1999;150(11):1152-1164).

\section{Enfermedades oportunistas en pacientes de sida al iniciarse el tratamiento antirretrovírico}

La morbilidad y mortalidad asociadas con la infección por el virus de la inmunodeficiencia humana (VIH) se han reducido enormemente gracias a la administración de medicamentos antirretrovíricos de gran eficacia. No obstante, siguen produciéndose enfermedades oportunistas y en la actualidad no están bien definidos los factores de riesgo que hacen que el enfermo empeore pese a la administración de fármacos potentes. Se sabe que el tratamiento se acompaña de una progresiva recuperación de la memoria inmunitaria, una restauración paulatina de las subpoblaciones de linfocitos T CD4 y una mayor respuesta proliferativa frente a la presencia de diferentes antígenos bacterianos y víricos. Pero la función inmunitaria pasa por un período de relativa latencia, especialmente en individuos con enfermedad avanzada que apenas inician el tratamiento. Es posible, además, que al empezar a administrarse el tratamiento con fármacos potentes se produzcan reacciones inflamatorias a los agentes patógenos oportunistas, probablemente debido a la rápida recuperación de la respuesta inmunitaria, y ello puede alterar el cuadro clínico.

Con objeto de examinar la incidencia de enfermedades oportunistas y sus factores de riesgo en pacientes tratados con antirretrovíricos potentes, se llevó a cabo un estudio poblacional prospectivo de cohorte en adultos con infección por el virus de la inmunodeficiencia humana (VIH). El estudio se efectuó en siete centros clínicos suizos y comprendió un total de 2410 personas, de las cuales 143 tuvieron enfermedades oportunistas después de iniciarse el tratamiento antirretrovírico con fármacos de gran potencia. La incidencia de estas infecciones se redujo de 15,1 por 100 años-persona en los 6 meses anteriores al tratamiento, a 7,7 en los 3 primeros meses del tratamiento, 2,6 en los 6 meses siguientes y 2,2 en el período de los 9 a 15 meses posteriores. Las reducciones de la incidencia mensual variaron de $38 \%$ en el caso del sarcoma de Kaposi $(P<0,001)$ a $5 \%$ en el del linfoma no Hodgkin $(P=$ 0,31 ). Los recuentos basales de células CD4 siguieron teniendo valor predictivo en cuanto a la progresión del cuadro clínico tras el inicio de los antirretrovíricos potentes. El riesgo relativo (RR) de contraer una enfermedad oportunista fue de 2,5 (IC95\%: 1,4 a 4,5) en personas con recuentos basales de 51 a $200 \times 10^{6} / \mathrm{L}$ y de $5,8($ IC95\%: 3,2 a 10,5$)$ en personas con recuentos basales menores de $51 \times$ $10^{6} / \mathrm{L}$. Independientemente de los recuentos basales de linfocitos T CD4, un aumento de estos linfocitos de $50 \times 10^{6} / \mathrm{L}$ o más y una concentración plasmática indetectable de ARN de VIH-1 $(<400$ copias $/ \mathrm{mL}$ ) al cabo del sexto mes se acompañaron de un riesgo reducido de problemas posteriores [RR de 0,32 (IC95\%: 0,20 a 0,52) y 0,39 (0,24 a 0,65), respectivamente].

Los datos aquí descritos indican que el riesgo de contraer una enfermedad oportunista en un paciente tratado con antirretrovíricos potentes es mayor durante los primeros meses del tratamiento. Los recuentos basales de linfocitos CD4 y la respuesta inmunológica y virológica al tratamieno fueron factores con valor predictivo en cuanto a la pro- 
gresión de la enfermedad. Los individuos con recuentos de células CD4 de $50 \times 10^{6} / \mathrm{L}$ o menos podrían necesitar una vigilancia clínica cercana al empezar el tratamiento intenso. (Lederberger B, Eggr M, Erard V, Weber R, Hirschel B, Furrer H, et al. AIDS-related opportunistic illnesses occurring after initiation of potent antiretroviral therapy: the Swiss HIV cohort study. JAMA 1999;282(23):2220-2226).

\section{El aceite de oliva confiere posible protección contra la artritis reumatoide}

La artritis reumatoide, enfermedad inflamatoria crónica, obedece a factores aún desconocidos, aunque se han postulado explicaciones de orden microbiológico, inmunológico, genético, hormonal y alimentario. En experimentos con ratas, la dieta se ha asociado con la aparición de poliartritis y hay pruebas cada vez más convincentes, basadas en estudios en seres humanos, de que una dieta a base de aceite de pescado o de oliva aminora los síntomas de artritis reumatoide, quizá por alteración de las respuestas inmunológica e inflamatoria. Un grupo de investigadores griegos se propuso estudiar la posibilidad de que el aceite de oliva, de uso general en Grecia, ejerza un efecto protector contra la artritis reumatoide. Con tal propósito examinó el consumo de más de 100 alimentos mediante la administración de un cuestionario validado. Se aplicó la prueba de ji al cuadrado para determinar la tendencia lineal y las razones de posibilidades (RP) relacionadas con el riesgo de padecer artritis reumatoide y el consumo de aceite de oliva, pescado, legumbres y una variedad de grupos alimentarios clasificados en cuartiles.

El riesgo de artritis reumatoide mostró una asociación inversa significativa solamente con el consumo de verduras cocidas (riesgo relativo (RR): $0,39)$ y aceite de oliva (RR: 0,39), según los resultados del análisis univariante. También se detectó una tendencia descendente significativa paralela al aumento del consumo de aceite de oliva (ji al cuadrado: 4,28; $P=0,03)$ y de legumbres cocidas (ji al cuadrado: 10,$48 ; P=0,001$ ). Los modelos de regresión logística múltiple confirmaron la presencia de una asociación inversa independiente entre el consumo de aceite de oliva y de verduras cocidas (razones de posibilidades (RP): 0,38 y 0,24 , respectivamente).

Los resultados de este estudio ponen de manifiesto la necesidad de estudiar más a fondo los mecanismos que explican la asociación entre el consumo de aceite de oliva y verduras cocidas y el riesgo de padecer artritis reumatoide. Entre ellos podrían figurar las propiedades antioxidantes de estos alimentos o su alto contenido de ácidos grasos n-9. (Linos A, Kaklamani VG, Kaklamani E, Kou- mantaki Y, Giziaki E, Papazoglou S, et al. Dietary factors in relation to rheumaotoid arthritis: a role for olive oil and cooked vegetables. Am J Clin Nutr 1999;70:1077-1082).

\section{Las enfermedades cardiovasculares: ¿cuándo empieza el peligro?}

La presencia de una asociación entre el riesgo de enfermedad cardiovascular y la estrechez económica en la vida adulta se ve respaldada por abundantes datos, pero pocos estudios han intentado averiguar si el riesgo también se vincula con la exposición a condiciones socioeconómicas adversas en edad temprana y durante los años que transcurren entre la niñez y la adultez. Según postula el modelo vitalicio, el riesgo de enfermedad cardiovascular obedece a una lenta acumulación de riesgos a lo largo de toda la vida, quizá de mayor intensidad durante ciertos períodos críticos en el desarrollo.

La mayor parte de los estudios efectuados hasta la fecha han destacado el alto valor predictivo de la posición socioeconómica en la niñez, que es independiente de la clase social alcanzada por el adulto; otros resaltan la influencia combinada de ambos factores. Con el doble propósito de 1) comparar la influencia ejercida por la posición socioeconómica en la niñez y la adultez en el riesgo de enfermedad cardiovascular y de 2) estimar el efecto de la clase socioeconómica alcanzada en la adultez habiendo hecho ajustes por las circunstancias infantiles, un grupo de investigadores escoceses efectuó un estudio transversal basado en la administración de un cuestionario sobre el estilo de vida en la infancia para determinar el índice relativo de desigualdad (relative index of inequality). La muestra se compuso de 4774 hombres y 2206 mujeres que participaban en el estudio longitudinal de Whitehall II y que habían nacido entre 1930 y 1953.

Los resultados revelaron una asociación inversa entre la posición socioeconómica del adulto (tipo de empleo) y varios factores de riesgo: el tabaquismo en el momento del estudio, la falta de actividad física en ratos de descanso, la razón peso: estatura, las concentraciones plasmáticas de lipoproteínas de alta densidad (HDL), triglicéridos, glucosa posprandial y fibrinógeno. Con la única excepción del tabaquismo en la mujer, fue más débil o nula la asociación entre estos factores y la posición socioeconómica en la niñez. En ambos sexos se detectó una asociación entre la posición socioeconómica en la infancia y el peso corporal del adulto; el tabaquismo en el momento del estudio; la razón cintura:estatura; y las concentraciones de HDL, colesterol y fibrinógeno en mujeres. La estatura, que es un reflejo de la salud constitucional, mostró una 
débil asociación con la clase social paterna y una asociación más fuerte con el tipo de empleo del adulto. El efecto combinado de adversidad socioeconómica en la niñez (clase paterna baja) y el desempeño por adultos de sexo masculino de trabajos de oficina de poca categoría se manifestó en un mayor índice de masa corporal $(P<0,001)$. Al hacer ajustes por la posición socioeconómica en edad temprana, teniendo en cuenta simultáneamente la clase social paterna y el grado de escolaridad del encuestado, no se alteró el efecto de la posición socioeconómica alcanzada en la adultez, salvo en el caso de una reducción del tabaquismo en la mujer.

En resumen, los factores de riesgo cardiovascular que se prestan a cuantificación en la edad adulta se asociaron más estrechamente, en términos generales, con la situación socioeconómica del adulto que con la del niño. Solamente en las mujeres se observó una fuerte asociación entre el hábito de fumar en la edad adulta y la clase social del padre. En ambos sexos el grado de obesidad mostró una asociación inversa con la posición socioeconómica en la niñez y también en la adultez. Tales resultados indican que la acumulación de un reservorio de "salud" y de factores de riesgo de enfermedad cardiovascular se inicia en la niñez y continúa en la edad adulta, bajo la influencia de factores socioeconómicos. (Brunner E, Shipley MJ, Blane D, Smith GD, Marmot MG. When does cardiovascular risk start? Past and present socioeconomic circumstances and risk factors in adulthood. J Epidemiol Community Health 1999;53(12):757-764).

\section{El tabaco, el alcohol y el riesgo de cáncer de las vías aerodigestivas superiores}

En países en desarrollo, los cánceres de las vías aerodigestivas superiores — cavidad bucal, faringe y laringe- ocupan el tercer lugar entre las neoplasias que afectan a los hombres y el cuarto entre las que afectan a las mujeres. Asimismo, estas neoplasias y la mortalidad que con ellas se asocia muestran una tendencia ascendente en casi todas partes. Se sabe desde hace mucho tiempo que el tabaco y el alcohol se asocian con el riesgo de este tipo de cáncer, junto con una dieta pobre en frutas y legumbres y otros factores de carácter ambiental. Incluso se postula la presencia de una interacción entre el alcohol y el cigarrillo, según la ubicación del tumor. Aunque se han efectuado estudios para determinar los riesgos atribuibles por separado al alcohol y al tabaco, la alta correlación entre ambos y el escaso número de sujetos de estudio que nunca han fumado o bebido han impedido llegar a conclusiones concretas. A esta dificultad se suma la del carácter multidimensional de estas variables, siendo escasos los estudios que han tenido en cuenta la intensidad y la duración de la exposición al tabaco y al alcohol.

Valiéndose de datos arrojados por un estudio de 784 casos hospitalizados y 1578 testigos (apareados según sexo, edad, período de admisión y zona de captación hospitalaria), un grupo de investigadores brasileños examinó el efecto combinado del consumo de alcohol y cigarrillos en el riesgo de sufrir cánceres escamocelulares de las vías aerodigestivas superiores. Los sujetos fueron entrevistados y sometidos a preguntas sobre sus hábitos de consumo de tabaco y alcohol y otras características. Se efectuó un análisis de regresión logística que reveló una interacción entre la exposición acumulada al tabaco y al alcohol y el riesgo de neoplasias escamocelulares de las vías aerodigestivas. Los efectos combinados del alcohol y el tabaquismo moderado en el riesgo de cánceres faríngeos excedieron los esperados con el modelo multiplicativo $(P=0,007)$. No obstante, no hubo confirmación estadística de una interacción con los cánceres de cavidad bucal $(P=0,28)$ o faringe $(P=0,95)$. Entre las personas que nunca habían fumado, las que habían sido fuertes bebedoras tuvieron un riesgo 9,2 veces mayor (IC95\%: 1,7 a 48,5) de sufrir un cáncer de la cavidad bucal, faringe y supraglotis que las que nunca habían bebido, observándose una tendencia ascendente en relación directa con la exposición acumulada $(P=0,013)$.

Los autores concluyen que hay poca uniformidad en la interacción que se produce entre el tabaco y el alcohol y los cánceres escamocelulares de faringe, y a la vez subrayan que el efecto depende del grado de exposición al tabaco. El alcohol puede tener un efecto promotor, pero también actúa como factor de riesgo independiente. (Schiecht NF, Franco EL, Pintos J, Hegassa A, Kowlaski LP, Oliveira $\mathrm{BV}$, et al. Interaction between tobacco and alcohol consumption and the risk of cancers of the upper aero-digestive tract in Brazil. 1999;150(11): 1129-1137).

\section{El tabaquismo del adulto y la depresión en niños y adolescentes: ¿se asocian?}

La conocida presencia de una relación entre la depresión y el hábito de fumar en personas adultas, respaldada por abundantes datos, ha llevado a algunos investigadores a preguntarse si la misma asociación se produce en la adolescencia y la niñez. En 1996, Patron et al. publicaron resultados confirmatorios que se basaron en un estudio de corte transversal en adolescentes. No obstante, faltaba explorar mediante estudios longitudinales la naturaleza temporal de la asociación observada entre el 
hábito de fumar y la depresión. Con este propósito, recientemente otros investigadores llevaron a cabo un estudio prospectivo basado en métodos de análisis de supervivencia y en datos procedentes de personas que oscilaban en edad entre la niñez tardía y mediados de la adolescencia. El estudio, que se basó en una muestra de 1731 estudiantes (de 8-9 y 13-14 años de edad), tuvo por objetivo determinar en qué medida el riesgo de empezar a fumar podría depender de un estado depresivo y viceversa. Los resultados, que se citan a continuación, arrojan nueva luz sobre la secuencia temporal entre el tabaquismo y la depresión en niños y adolescentes.

El tabaquismo se asoció con un aumento moderado del riesgo de sufrir un primer episodio depresivo (riesgo relativo sin ajuste (RRSA): 1,73; IC95\%: 1,35 a 2,21; $P<0,001)$. No se observaron diferencias en función del sexo, pero los sujetos de raza blanca no hispanos tuvieron un riesgo de sufrir depresión ligeramente menor que los de otras razas (RRSA: 1,35 ; IC95\%: 1,10 a 1,66; $P=0,004$ ). Los ajustes por variables covariantes tuvieron poco efecto en las estimaciones (riesgo relativo ajustado (RRA) asociado con el tabaquismo: 1,66; IC95\%: 1,28 a 2,16; $P<$ $0,001)$. El haber sido bebedor anteriormente no mostró ninguna asociación con un mayor riesgo de sufrir depresión (RRA: 1,19; IC95\%: 0,99 a 1,42; $P=0,059$ ).

No se detectó ninguna asociación entre la presencia de antecedentes de depresión y el inicio pos- terior del hábito de fumar (RRNA: 1,07); IC95\%: 0,76 a 1,52; $P=0,690)$. Tampoco se observó asociación alguna entre el sexo del sujeto y el inicio del hábito de fumar en el grupo de edad estudiado, pero los jóvenes depresivos que no eran de raza blanca sí mostraron un menor riesgo de empezar a fumar que los blancos no hispanos que sufrían depresión (RRNA: 0,68; IC95\%: 0,50 a 0,94; $P=0,019$ ). El consumo previo de alcohol se asoció con un riesgo aumentado de empezar a fumar (RRNA: 2,60; IC95\%: 1,96 a 3,47; $P<0,001)$, aun después de hacer ajustes por la presencia de factores covariantes.

El estudio aquí descrito tuvo limitaciones importantes: 1) la muestra provino de una zona urbana del litoral nororiental de Estados Unidos y no es representativa de toda la población; 2) los resultados no pueden compararse con los de estudios en adultos debido al enfoque exclusivo en la depresión en la juventud; 3) los datos obtenidos derivan de las respuestas dadas por los propios jóvenes a un cuestionario y están sujetos a sesgos de recordación e interpretación; 4) con los años, muchos jóvenes abandonaron el estudio. No obstante, los resultados permiten concluir que en el grupo de edad estudiado hay un posible vínculo causal entre el hábito de fumar y la presencia posterior de depresión, pero no viceversa. ( $\mathrm{Wu} \mathrm{L-T,} \mathrm{Anthony} \mathrm{JC.} \mathrm{Tobacco}$ smoking and depressed mood in late childhood and early adolescence. 1999;89(12):1837-1840). 